\section{Entomofauna of Kane Wildlife Sanctuary, Arunachal Pradesh, northeastern India}

\author{
O. Tarunkumar Singh ${ }^{1}$, J. Chakravorty ${ }^{2} \&$ \\ R. Varatharajan ${ }^{3}$
}

${ }^{1}$ Centre of Biodiversity, ${ }^{2}$ Departments of Zoology, Rajiv Gandhi University, Itanagar, Arunachal Pradesh 791112, India ${ }^{3}$ Life Sciences Department, Manipur University, Imphal, Manipur 795003, India

Email: ${ }^{1}$ oinamba2010@rediffmail.com

Studies on the entomofauna of Arunachal Pradesh, northeastern India are referable to the works of Mitra (1976), Tandon \& Khera (1978), Lahiri (1979 a,b), Basu \& Sengupta (1980), Chatterjee \& Saha (1981), Sengupta \& Sengupta (1981), Biswas \& Biswas (1985), Biswas \& Chatterjee (1985), Ghosh (1985), Bhargava (1989, 1996), Pal $(1985,1992 a, 1992 b, 1992 c, 1993)$ and Chakrabarty et al. (1994) in which nearly 600 species of insects belonging to Collembola, Odonata, Orthoptera, Blattaria, Coleoptera, Diptera, Hymenoptera and Hemiptera have been reported. Later, Ramakrishna \& Alfred (2006) compiled all the reported species from Arunachal Pradesh

Date of publication (online): 26 December 2010

Date of publication (print): 26 December 2010

ISSN 0974-7907 (online) | 0974-7893 (print)

Editor: Kailash Chandra

Manuscript details:

Ms \# 01946

Received 15 February 2008

Final received 02 November 2010

Finally accepted 18 November 2010

Citation: Singh, O.T., J. Chakravorty \& R. Varatharajan (2010) Entomofauna of Kane Wildlife Sanctuary, Arunachal Pradesh, northeastern India. Journal of Threatened Taxa 2(13): 1392-1400.

Copyright: (c) O. Tarunkumar Singh, J. Chakravorty \& R. Varatharajan 2010. Creative Commons Attribution 3.0 Unported License. JoTT allows unrestricted use of this article in any medium for non-profit purposes reproduction and distribution by providing adequate credit to the authors and the source of publication.

Acknowledgements: The authors are thankful to Department of Zoology and Centre of Biodiversity, Rajiv Gandhi University, Itanagar, for the facilities. The first author is thankful to the University Grants Commission, New Delhi for the financial support through Dr. D.S. Kothar Post Doctoral Fellowship. Thanks are also due to Mr. M.B. Lal, Principal Chief Conservator of Forest, Government of Arunachal Pradesh, fo prior permission to carry out the survey. Our appreciations are due to Mr. Tayeng Mize (DFO), Kane Wildlife Sanctuary and subordinate staffs for their tireless help during the survey trips. Thanks are also extended to Director, SFRI (Itanagar) and Dr. G. Srinivasan, Scientists-in-charge ZSI (Itanagar) for providing necessary assistances in insect identification. Our gratefulness are extended to Dr. Ch. Chitrpati Devi, Lecturer, D.M. College, Imphal for determining the identities of Collembolan insects.

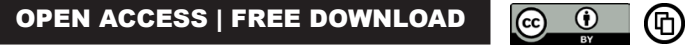

in the State Fauna series. Detailed systematic accounts were given in the state faunal series - 13 (part-II) of the Zoological Survey of India (Anonymous 2006b). It included 645 species of insects under 320 genera and 44 families in which 119 species were new records for the state. Recently, Singh et al. (2007) recorded 100 species of Coleoptera from various habitats of Arunachal Pradesh, in which 25 species under 20 genera were new to the state. As the state is one of the global biodiversity hot spots (Myer et al. 2000) and is endowed with rich flora and fauna (Das 2006), intensive studies are needed at the earliest to document all of the insects.

Kane Wildlife Sanctuary is located in the West Siang District of Arunachal Pradesh between $27^{\circ} 35^{\prime}-27^{\circ} 45^{\prime} \mathrm{N}$ \& $94^{\circ} 35^{\prime}-94^{\circ} 45^{\prime} E$. Spread over an area of $55 \mathrm{~km}^{2}$, it is bounded by the Siri River on the north and northwest. Along Likabali Road $(15 \mathrm{~km})$ on the east, Nilakh Nallah (hill stream) on the south and Bardam Modi (hill range) on the south west (Anonymous 2007). The area falls in the Eastern Himalayan bio-geographical zone with montane evergreen tropical and subtropical forest vegetation (Singh et al. 2002). The Sanctuary is unique in having a wide range of surface elevation $(200-2000 \mathrm{~m})$, varied temperature fluctuations $\left(5-38{ }^{\circ} \mathrm{C}\right)$ at different altitudinal levels with relatively high humidity and excessive rainfall (>3000 mm/year). The area has been declared protected as a sanctuary under the Wildlife Protection Act, 1972 for the conservation of elephants, small cats and deer along with their associated flora and fauna since 1992. However, little has been known about its flora and fauna till today. Therefore, the present study was taken up as an initiative to identify and document its faunal resources, particularly insects. The study assumes significance with reference to the conservation of entomofauna of the region.

Materials and Methods: Insect samples have been collected and preserved for study following Ghosh \& Sengupta (1982). In the case of collembolans and litter thrips, the fallen leaves, soil and humus from all possible habitats were collected at random in polythene bags. The samples were subjected to the process of extraction by using modified Tullgren funnel extractors and collected specimens were preserved in $70 \%$ alcohol (Ananthakrishnan 1984). Collections were made for a period of one year (2007) with frequent trips during January to April and August to November. Collections were made within 200-1500 m altitudinal range covering nearly $15 \mathrm{~km}^{2}$ deep inside the eastern portion of the sanctuary on the Likabali side starting from Kane Village. Collected specimens were sorted out to species level and identified using valid key characters and by comparison with the materials available in the Insect Diversity Unit (RGU), Manipur University, ZSI and SFRI (Itanagar). Taxonomic publications including Anonymous (1999, 2003, 2006b), 
Borowiec (2001), Ghate et al. (2003), Subramanian (2005), Mitra (2006) have been referred to for the correct determination of the identities of the insects. Distributions of the identified species within Arunachal Pradesh were compiled based on Sen et al. (1988), Chakravorty et al. (1994), and Anonymous (2006 a,b).

Results and Discussion: During the one year preliminary survey, 170 species of insects under nine orders were identified and are listed along with their distributions in Table 1. This included 70 species of Coleoptera (beetles), 23 species each of Odonata (dragonflies) and Thysanoptera (thrips), 18 species of Orthoptera (grasshoppers), 13 species of Hymenoptera (bees), eight species of Collembola (springtails), seven species of Diptera (flies), five species of Hemiptera (bugs) and two species of Dictyoptera (mantis). Of these 39 species are new records for the state.

Coleopteran fauna were dominant in the present collection. Beetles being the largest among the insect orders are associated with various habitats in the forest ecosystem. The majority of them are phytophagous and scavengers inhabiting various plants, debris and animal excreta. Phytophagous beetles play an important role in checking the populations of many other insects including harmful insects such as aphids, coccids, aleuryds, plantmites and other foliage feeders. Scavengers, being associated with debris and animal excreta act as detritivores, thereby helping in breaking down plant and animal remains that contribute to the recycling of nutrients and the cleaning of the environment.

The present collection reveals the occurrence of 23 species of Thysanoptera (Thrips). Among them three species viz., Elaphrothrips spiniceps, Nesothrips lativentris and Xylaplothrips debilis are mycophagous forms feeding on fungal mycelia. Of the phytophagous forms Gigantothrips elegans, Liothrips ananthakrishnani and Liothrips sp. are found to cause structural abnormalities in their respective host plants. The first one causes damage to the ficus leaf and the latter two are found to form leaf rolling galls of Acanthus sp. and Pozoulzia zeylanica respectively. Again two terebrantian species, Panchaetothrips indicus and Sciothrips cardamomi, which were collected from wild herbs during the survey.

The Orthopteran collection contained 18 species belonging to six families. Among them 11 species were under the family Acrididae, two species each under Tettigoniidae, Schizodactylidae and Gryllidae, respectively. The family Pyrgomorphidae was represented by a single species Tagasta indica. The two species of Schizodactylidae, Schizodactylus monstrosus and $S$. tuberculatus were frequently encountered within sandy burrows near the hillsteams and moist foothills.

Collembolan fauna included eight species under two families, Entomobryidae and Hypogastruridae. By virtue of their association with litter and soil, these tiny apterous insects are known for their role in recycling the forest nutrients. Dipteran and Hemiptera were represented by seven and five species respectively. In addition to the above collections, different species of butterflies, moths and a half dozen Cicadas were also collected during the study. The identities of these insects are yet to be determined.

Conclusion: Being a short term preliminary study coupled with mountainous moist habitats of the sanctuary, only limited areas could be covered during the survey. However, the above observations indicate that Kane Wildlife Sanctuary is a rich spot for entomological fauna. It also suggests that a long term survey covering maximum habitats over different seasons would be required at the earliest to explore and document the entomological wealth of the protected area.

\section{REFERENCES}

Ananthakrishnan, T.N. (1984). Bioecoology of Thrips. Indira Publishing House, Michigan, USA, 233pp.

Anonymous (1999). Fauna of Meghalaya. State fauna Series 4(Part V). Zoological Survey of India, Kolkata Publication, $666 \mathrm{pp}$

Anonymous (2003). Fauna of Sikkim. State fauna Series 9 (Part III). Zoological Survey of India, Kolkata Publication, 411pp.

Anonymous (2006a). Fauna of Arunachal Pradesh. State fauna Series 13 (Part I). Zoological Survey of India Publication, $666 \mathrm{pp}$.

Anonymous (2006b). Fauna of Arunachal Pradesh. State fauna Series 13 (Part II). Zoological Survey of India Publication, $518 p p$.

Anonymous (2007). The Arunachal Pradesh Gazette (Part I). Government of Arunachal Pradesh Publication XVIII: 57-58.

Basu, C.R. \& T. Sengupta (1980). On a new genus and a new species of Alticinae (Coleoptera: Chrysomelidae) from Arunachal Pradesh. Records of Zoological Survey of India 76(1-4): 143-146.

Bhargava, R.N. (1989). On a collection of Odonata from west Kameng District, Arunachal Pradesh. Indian Odonatol 2: 4546.

Bhargava, R.N. (1996). A new mole cricket of the genus Scapteriscus (Orthoptera: Gryllotalpidae) from Arunachal Pradesh, India. Entomon 21(2): 191-193.

Biswas, D.N. \& S. Biswas (1985). Insecta: Coleoptera: Staphylinidae. On a collection of Staphylinidae (Insecta: Coleoptera) from Namdapha Wild Life Sanctuary, Tirap district, Arunachal Pradesh, India. Records of Zoological Survey of India 82(1-4): 139-146.

Biswas, S. \& S.K. Chatterjee (1985). Insects: Scarabaeidae: Coprinae. Records of Zoological Survey of India 82(1-4): 147-177.

Borowiec, L. (2001). New records of Asian and Australopapuan Cassidinae with a description of five new species of Cassida L. from Thailand (Coleoptera: Chrysomelidae: Cassidinae). Genus 12(4). Wroclaw 28 XII: 493-562.

Chakrabarty, S.P., L.K. Ghosh \& R.C. Basu (1994). On a collection of Hemiptera from Namdapha Biosphere reserve in Arunachal Pradesh. Records of Zoological Survey of India. Occasional paper 161: 1-38.

Chatterjee, S.K. \& G.N. Saha (1981). On a collection of Scarabaeidae (Insecta: Coleoptera) from Arunachal Pradesh with the description of a new species. Records of Zoological Survey of India 78(1-4): 155-167.

Das, A. K. (2006). Biodiversity Conservation, pp. 160-177. In: 
Table 1. List of Insects collected from Kane Wildlife Sanctuary

\begin{tabular}{|c|c|c|c|}
\hline & Species & Common name & Distribution / new record to Arunachal Pradesh \\
\hline & Order: Coleoptera & & \\
\hline & Family: Passalidae & & \\
\hline 1 & Acerais helferi Kuw. & Bess Beetle & New record \\
\hline \multirow[t]{2}{*}{2} & Aulococyclus sp. & $\begin{array}{l}\text { Patent Leather } \\
\text { Beetle / Bess Beetle }\end{array}$ & East Siang, Kane (W. Siang) \\
\hline & Family: Chrysomelidae & & \\
\hline 3 & Agrosteomela fallaciosa Stal & Leaf Beetle & New record \\
\hline 4 & Agrosteomela indica (Hope) & Leaf Beetle & Subansiri, Kurungkumey, Dibang Valley, Kane (West Siang) \\
\hline 5 & Aplosonyx scutellatus (Baly) & Fringed-toed Lizard & Changlang, Dibang Valley, East Kameng, Kane (W. Siang) \\
\hline 6 & Aspidomorpha dorsata (Fabricius) & Tortoise Beetle & New record \\
\hline 7 & Aspidomorpha indica Boheman & Tortoise Beetle & Subansiri, West Kameng, Kane (W. Siang) \\
\hline 8 & Aspidomorpha sanctaecrucis (F) & Tortoise Beetle & East Siang, Changlang, Likabali \& Kane (W. Siang) \\
\hline 9 & Aulacophora almora Maulik & Leaf Beetle & Dibang Valley, Changlang, Kurungkumey, Dibang Valley, Kane (W. Siang) \\
\hline 10 & Aulacophora bicolor (Weber) & Leaf Beetle & East Siang, Upper Siang, Papumpare, Changlang, Kane (W. Siang) \\
\hline 11 & Aulacophora foveicollis ( Lucas) & Red Pumpkin Beetle & East Kameng, Subansiri, East Siang, Kane (W. Siang) \\
\hline 12 & Aulacophora lewisii Baly & Pumpkin Beetle & New Record \\
\hline 13 & Basiprinota maculipennis (Boheman) & Leaf Beetle & Changlang, Dibang Valley, Papumpare, Kane (W. Siang) \\
\hline 14 & Cassida occursans Spaeth & Tortoise Beetle & Changlang, Subansiri, East Siang, Likabali \&Kane (W. Siang) \\
\hline 15 & Chrysomela populi Linn. & Leaf Beetle & New record \\
\hline 16 & Cneorane rubicollis (Hope) & Leaf Beetle & West Kameng, Subansiri, East Siang, Changlang, Kane (W. Siang) \\
\hline 17 & Cryptocephalus exsulans Suffrian & Leaf Beetle & Likabali, Kane (W. Siang) \\
\hline 18 & Galerucella grisescens (Joannis) & Leaf Beetle & New record \\
\hline 19 & Haplosoma unicolor (Illiger) & Leaf Beetle & Papumpare, Changlang, Subansiri, Kane (W. Siang) \\
\hline 20 & Humba cyanicollis (Hope) & Leaf Beetle & East Kameng, West Kameng, Dibang Valley, Likabali \& Kane (W. Siang) \\
\hline 21 & Lema nigricollis Jacoby & Leaf Beetle & Subansiri, Changlang, East Kameng, Kane (W. Siang) \\
\hline 22 & Meristata dohrni ( Baly) & Leaf Beetle & Subansiri, Changlang, Kane (W. Siang) \\
\hline 23 & Monolepta signata (Olivier) & Cucumber Beetle & Subansiri, Lohit, West Kameng, Kane (W. Siang) \\
\hline 24 & Oides livia (Fabricius) & Beetle & Eastkameng, West Kameng Kurungkumey, Kane (W. Siang) \\
\hline 25 & Oides maculate (Olivier) & Beetle & New record \\
\hline 26 & Periclitena vigorsi (Hope) & Beetle & East Kameng, Dibang Valley, Kane (W. Siang) \\
\hline 27 & Pseudadimonia variolosa (Hope) & Beetle & New record \\
\hline 28 & Sphenoraia (S) bicolor (Hope) & Beetle & New record \\
\hline \multirow[t]{2}{*}{29} & Xuthea orientalis Baly & Beetle & Changlang, East Kameng, Kane (W. Siang) \\
\hline & Family: Scarabaeidae & & \\
\hline 30 & Anomala dimidiate (Hope) & White Grub & New record \\
\hline 31 & Anomala sp. & White Grub & East Siang, Kane (W. Siang) \\
\hline 32 & Copris corpulentus Gillet & Dung Beetle & East Siang, Upper Siang, Papumpare, Changlang, Kane (W. Siang) \\
\hline 33 & Copris indicus Gillet & Dung Beetle & East Siang, Papumpare, Dibang Valley, Tawang, Along \& Kane (W. Siang) \\
\hline 34 & Copris magicus Harold & Dung Beetle & East Siang, Upper Siang, Kane (W. Siang) \\
\hline 35 & Copris $(P)$ punctatus Wiedeman & Dung Beetle & Tawang, Namdapha, Changlang, Kane (W. Siang) \\
\hline 36 & Copris siangensis Biswas & Dung Beetle & Lower Subansiri, East Siang, Upper Siang, Pasighat \& Kane (W. Siang) \\
\hline 37 & Onitis castaneous Redtenbacher & Dung Beetle & East Siang, Kane (W. Siang) \\
\hline
\end{tabular}




\begin{tabular}{|c|c|c|c|}
\hline & Species & Common name & Distribution / new record to Arunachal Pradesh \\
\hline 38 & Onitis falcatus (Wulfen) & Dung Beetle & New record \\
\hline 39 & Onitis feae Felsche & Dung Beetle & East Siang, Kane (W. Siang) \\
\hline 40 & Onitis subopacus Arrow & Dung Beetle & East Siang, Kane (W. Siang) \\
\hline 41 & Oryctes rhinoceros ( L) & Rhinoceros Beetle & New record \\
\hline \multirow[t]{2}{*}{42} & Rhynchophorus ferrugineus (Olivier) & Red Palm Beetle & New record \\
\hline & Family: Cerambycidae & & \\
\hline 43 & Anoplophora sp. & Long-horned Beetle & East Siang, Kane (W. Siang) \\
\hline 44 & Batocera numitor (Newman) & Longicorn Beetle & New record \\
\hline 45 & Batocera parryi (Hope) & Longicorn Beetle & East Siang, Kane (W. Siang) \\
\hline 46 & Hoplocerambyx spinicornis Newman & Borer Beetle & New record \\
\hline 47 & Stromatium barbatum (Fab.) & Kulsi Teak Borer & Along, Likabali \& Kane (W. Siang) \\
\hline 48 & Stromatium sp. & Forest Longicorn & East Siang, Kane (W. Siang) \\
\hline 49 & Xylotrechus quadripes Chevr. & $\begin{array}{l}\text { Coffee White Stem } \\
\text { Borer }\end{array}$ & East Siang, Papumpare, Kane (W. Siang) \\
\hline \multirow[t]{2}{*}{50} & Xylotrechus smei (Lap.et.Gory.) & Long-horned Beetle & East Siang, Papumpare, Kane (W. Siang) \\
\hline & Family: Carabidae & & \\
\hline 51 & Catascopus micabilis Bates & Ground Beetle & East Siang, Papumpare, Dibang Valley, Tawang, Along \& Kane (W. Siang) \\
\hline 52 & Ophionea indica (Thunb) & Carabid Beetle & East Siang, Papumpare, Subansiri, Kane (W. Siang) \\
\hline \multirow[t]{2}{*}{53} & Simous nigriceps (Weid) & Ground Beetle & East Siang, Papumpare, Changlang, Kane (W. Siang) \\
\hline & Family: Lucanidae & & \\
\hline 54 & Calodes cuvera Hope & Stag Horn Beetle & New record \\
\hline 55 & Calodes siva Hope \& Wetson & Stag Horn Beetle & New record \\
\hline 56 & Lucanus cantori Hope & Giant Stag Beetle & New record \\
\hline \multirow[t]{2}{*}{57} & Lucanus laminifer Waterhouse & Giant Stag Beetle & New record \\
\hline & Family: Curculionidae & & \\
\hline 58 & Cyrtotrachelus longimanus $\mathrm{F}$ & Bamboo Weevil & New record \\
\hline \multirow[t]{2}{*}{59} & $\begin{array}{l}\text { Cyrtotrachelus rufopectinipes } \\
\text { Chevrolet. }\end{array}$ & $\begin{array}{l}\text { Snout Beetle / Stem } \\
\text { Weevil }\end{array}$ & New record \\
\hline & Family: Histeridae & & \\
\hline 60 & Hypocaccus sinae (Marseul) & Steel Beetle & Papumpare, East Kameng, East Siang, Kurungkume, Kane (W. Siang) \\
\hline 61 & Pachylister chinensis (Quensel) & Hister Beetle & Changlang, Kurungkume, East Siang, Kane (W. Siang) \\
\hline 62 & Platylister strialis (Marseul) & Steel Beetle & East Siang, Kane (W. Siang) \\
\hline \multirow[t]{2}{*}{63} & Platylomalus teibodae (Marseul) & Steel Beetle & East Siang, Changlang, Kane (W. Siang) \\
\hline & Family: Cucujidae & & \\
\hline 64 & Laemophloes neglectus Grouvelle & Paper Flate Beetle & Papumpare, Subansiri, Kane (W. Siang) \\
\hline \multirow[t]{2}{*}{65} & Placonotus proximus Grouvelle & Paper Flate Beetle & Papumpare, East Siang, Subansiri, Kane (W. Siang) \\
\hline & Family: Staphylinidae & & \\
\hline \multirow[t]{2}{*}{66} & Leptochirus (S)laevis Castelnau & Rove Beetle & Papumpare, Kane (W. Siang) \\
\hline & Family: Meloidae & & \\
\hline 67 & Mylabris cichorii (Linn.) & $\begin{array}{l}\text { Flower Beetle / } \\
\text { Blister Beetle }\end{array}$ & Papumpare, East Siang, Changlang, Kane (W. Siang) \\
\hline 68 & Mylabris himalayaensis Saha & Flower Beetle & Papumpare, East Siang, Along \& Kane (W. Siang) \\
\hline
\end{tabular}




\begin{tabular}{|c|c|c|c|}
\hline & Species & Common name & Distribution / new record to Arunachal Pradesh \\
\hline & Family: Silvanidae & & \\
\hline 69 & Protosilvanus lateritius (Reitter) & Silvanid Beetle & East Kameng, Subansiri, Dibang Valley, Changlang, Kane (W. Siang) \\
\hline \multirow[t]{3}{*}{70} & $\begin{array}{l}\text { Psammoecus trimaculatus } \\
\text { Motschulsky }\end{array}$ & Silvanid Beetle & Subansiri, Dibang Valley, Changlang, Papumpare, Kane (W. Siang) \\
\hline & Order: Collembola & & \\
\hline & Family: Entomobryidae & & \\
\hline 71 & Callyntrura japonica (Kinoshita) & $\begin{array}{l}\text { Entomobryid /Spring } \\
\text { Tail }\end{array}$ & Subansiri, West Kameng, Kane (W. Siang) \\
\hline 72 & Dicranocentroides fasciculatus Imms & Spring Tail & Papumpare, West Kameng, Kane (W. Siang) \\
\hline 73 & Dicranocentroides salmoni Mitra & Spring Tail & Papumpare, Likabali And Kane (W. Siang) \\
\hline 74 & Homodia cingula Borner & Spring Tail & Subansiri, Kane (W. Siang) \\
\hline 75 & Lepidosira unguserrata Salmon & Spring Tail & Subansiri, Tawang, Kane (W. Siang) \\
\hline 76 & Lobella kraepelini Yosii & Spring Tail & West Kameng, Kane (W. Siang) \\
\hline \multirow[t]{2}{*}{77} & Salina indica (Imms) & Spring Tail & Papumpare, East Siang, Kane (W. Siang) \\
\hline & Family: Hypogastruridae & & \\
\hline \multirow[t]{3}{*}{78} & Hypogastrura indovari Salmon & Gun Powder Mite & Papumpare, Tawang, Basar \& Kane (W.Siang) \\
\hline & Order: Dictyoptera & & \\
\hline & Family: Blaberidae & & \\
\hline \multirow[t]{2}{*}{79} & Cryptocera sp. & Wood Roaches & East Siang, Kane (W. Siang) \\
\hline & Family:Mantidae & & \\
\hline \multirow[t]{3}{*}{80} & Mantis sp. & Preying Mantis & East Siang, Kane (W. Siang) \\
\hline & Order: Diptera & & \\
\hline & Family:Sargophagidae & & \\
\hline \multirow[t]{2}{*}{81} & Bercaea cruentata (Meigen) & Flesh Fly & East\&West Kameng, Lower \& Upper Subansiri, Kane (W. Siang) \\
\hline & Family: Calliphoridae & & \\
\hline 82 & $\begin{array}{l}\text { Calliphora(Calliphora)vicina } \\
\text { Robineau Desvoidy }\end{array}$ & Blue Bottle/ Blow Fly & West Kameng, Kane (W. Siang) \\
\hline \multirow[t]{2}{*}{83} & $\begin{array}{l}\text { Crysomya megacephala } \\
\text { ( Fabricius) }\end{array}$ & Oriental Latrine Fly & East \& West Kameng, Kane (W. Siang) \\
\hline & Family: Bombyliidae & & \\
\hline 84 & Ligyra oenomaus (Rondani) & Bee Fly & West Kameng, Kane (W. Siang) \\
\hline \multirow[t]{2}{*}{85} & Ligyra semifuscata (Brunetti) & Bee Fly & East Siang, Daporizo, Kane (W. Siang) \\
\hline & Family: Conopidae & & \\
\hline \multirow[t]{2}{*}{86} & Physocephala tenella Bigot & Thick-headed Flies & Along \& Kane (W. Siang) \\
\hline & Family: Tabanidae & & \\
\hline \multirow[t]{3}{*}{87} & Tabanus fuscomaculatus Ricardo & Horse Flies & East Siang, Kane (W. Siang) \\
\hline & Order: Hemiptera & & \\
\hline & Family:Pentatomidae & & \\
\hline 88 & Dalpada jugatoria Lethriery & Stink Bug & Tirap, Changlang, Kane (W. Siang) \\
\hline 89 & Nezara viridula (Linn.) & Green Stink Bug & New record \\
\hline \multirow[t]{2}{*}{90} & Pentatomid sp. & $\begin{array}{l}\text { Forest Bug/ True } \\
\text { Bug }\end{array}$ & East Siang, Kane (W. Siang) \\
\hline & Family: Coreidae & & \\
\hline 91 & Leptocorisa acuta (Thunberg) & Rice Seed Bug & Namdapha (Changlang), Kane (W.Siang) \\
\hline
\end{tabular}




\begin{tabular}{|c|c|c|c|}
\hline & Species & Common name & Distribution / new record to Arunachal Pradesh \\
\hline \multirow[t]{3}{*}{92} & Notobitus excellens Distant & Squash Bug & Tirap, Namdapha (Changlang), Kane (W.Siang) \\
\hline & Order: Hymenoptera & & \\
\hline & Family: Apidae & & \\
\hline 93 & Apis (Megapis) dorsata (Fabricius) & Rock Bee & Kameng, Subansiri, Papumpare, Kane (W. Siang) \\
\hline 94 & Apis indica Fabricius & Indian Honey Bee & East and West Kameng, Papumpare, Tawang, Subansiri, Kane (W. Siang) \\
\hline 95 & Apis (Micrapis) florea Fabricius. & Little Bee & Changlang, West Kameng, Kane (W. Siang) \\
\hline \multirow[t]{2}{*}{96} & Bombus orientalis Smith & Bumble Bee & Subansiri, West Kameng, Kane (W. Siang) \\
\hline & Family: Vespidae & & \\
\hline 97 & Parapolybia indica (Saussure) & Paper Wasps & West Kameng, East Siang, Kane (W. Siang) \\
\hline 98 & $\begin{array}{l}\text { Polistes (Nygmopolistes) } \\
\text { tenebricosus sulcatus Smith }\end{array}$ & Wasps & Changlang, Kane (W. Siang) \\
\hline 99 & $\begin{array}{l}\text { Polistes (Megapolistes) olivaceus } \\
\text { (De Geer) }\end{array}$ & Yellow Wasps & Subansiri, West Kameng, Changlang, Kane (W. Siang) \\
\hline 100 & Vespa basalis Smith & Black-bellied Hornet & $\begin{array}{l}\text { East Kameng, West Kameng, Changlang, East Siang, Likabali \& Kane } \\
\text { (W. Siang) }\end{array}$ \\
\hline 101 & Vespa bicolor Fabricius & Common Wasps & Subansiri, East Kameng, West Kameng, Likabali \& Kane (W. Siang) \\
\hline 102 & Vespa mandarinia magnifica Smith & Hornet & East Kameng, West Kameng, East Siang, Kane (W. Siang) \\
\hline \multirow[t]{2}{*}{103} & Vespa tropica ducalis Smith & Hornet & Subansiri, East Siang, Kane (W. Siang) \\
\hline & Family: Anthophoridae & & \\
\hline 104 & Xylocopa latipes (Drury) & Black Carpenter & New record \\
\hline \multirow[t]{3}{*}{105} & Xylocopa pubescens (Spinola) & Carpenter Bee & New record \\
\hline & Order: Odonata & & \\
\hline & Family: Coenagrionodae & & \\
\hline 106 & Aciagrion pallidum Selys & $\begin{array}{l}\text { Narrow-winged } \\
\text { Damselfly }\end{array}$ & Kameng, Dibang Valley, Kane (W. Siang) \\
\hline 107 & Agriocnemis pygmaea (Rambur) & Pigmy Wisp & Kameng, Dirang, East Siang, Kane (W. Siang) \\
\hline 108 & $\begin{array}{l}\text { Ceriagrion fallax cerinomelas } \\
\text { Lieftinck }\end{array}$ & $\begin{array}{l}\text { Narrow-winged } \\
\text { Damselfly }\end{array}$ & Subansiri, Kameng, Kane (W. Siang) \\
\hline \multirow[t]{2}{*}{109} & Pseudagrion australasiae (Selys) & Blue Damselfly & Subansiri, Kameng, East Siang, Kane (W. Siang) \\
\hline & Family: Euphaeidae & & \\
\hline \multirow[t]{2}{*}{110} & Anisopleura lestoides( Selys) & Damselfly & East Siang, Kane (W. Siang) \\
\hline & Family: Platycnemididae & & \\
\hline 111 & Calicnemia eximia (Selys) & Scarlet Openwing & Namdapha ( Changlang), Kane (W. Siang) \\
\hline \multirow[t]{2}{*}{112} & Copera vittata serapica (Selys) & $\begin{array}{l}\text { Weak Flier/ } \\
\text { Damselfly }\end{array}$ & East Siang, Changlang, Kane (W. Siang) \\
\hline & Family: Libellulidae & & \\
\hline 113 & Diplacodes trivialis (Rambur) & Ground Skimmer & Changlang, West Kameng, Likabali \& Kane (W. Siang) \\
\hline 114 & Neurothemis fulvia (Drury) & $\begin{array}{l}\text { Fulvous Forest } \\
\text { Skimmer }\end{array}$ & Tirap, Subansiri, East Siang, Kane (W. Siang) \\
\hline 115 & Orthetrum anceps (Schneider) & Marsh Hawk & East Siang, Kane (W. Siang) \\
\hline 116 & Orthetrum brunneum Fouscolombe & Marsh Hawk & Subansiri, East Siang, West Siang \\
\hline 117 & Orthetrum glaucum (Brauer) & Blue Marsh Hawk & Subansiri, Namdapha (Changlang), Kane (W. Siang) \\
\hline 118 & Pantala flavescens (Fabricius) & Wandering Gliders & East Siang, West Siang \\
\hline 119 & Tholymis tillarga (Fabricius) & $\begin{array}{l}\text { Coral-tailed Cloud } \\
\text { Wing }\end{array}$ & Likabali and Kane (W. Siang) \\
\hline
\end{tabular}




\begin{tabular}{|c|c|c|c|}
\hline & Species & Common name & Distribution / new record to Arunachal Pradesh \\
\hline & Family: Gomphidae & & \\
\hline 120 & Ictinogomphus rapax (Rambur) & Common Club Tail & East Siang, West Siang \\
\hline 121 & Onychogomphus biforceps ( Selys) & Club-tailed Dragonfly & East Siang, West Siang \\
\hline 122 & $\begin{array}{l}\text { Onychogomphus cacharicus } \\
\text { (Fraser) }\end{array}$ & Club-tailed Dragonfly & East Siang, West Siang \\
\hline 123 & Paragomphus lineatus (Selys) & Club-tailed Dragonfly & East Siang, Kane (W. Siang) \\
\hline \multirow[t]{2}{*}{124} & $\begin{array}{l}\text { Phaenandrogomphus aureus } \\
\text { (Laidlaw) }\end{array}$ & Club-tailed Dragonfly & Subansiri, East Siang, Kane (W. Siang) \\
\hline & Family: Calopterygidae & & \\
\hline 125 & Matrona basilaris basilaris Selys & $\begin{array}{l}\text { Broad-winged } \\
\text { Damselfly }\end{array}$ & East Siang, Kane (W. Siang) \\
\hline \multirow[t]{2}{*}{126} & $\begin{array}{l}\text { Neurobasis chinensis chinensis } \\
\text { (Linn.) }\end{array}$ & $\begin{array}{l}\text { Metallic Green } \\
\text { Damselfly }\end{array}$ & Subansiri, Tirap, Kane (W. Siang) \\
\hline & Family: Chlorocyphidae & & \\
\hline 127 & Rhinocypha ignipennis Selys & $\begin{array}{l}\text { Broad-winged } \\
\text { Damselfly }\end{array}$ & Subansiri, West Kameng, East Siang, West Siang \\
\hline \multirow[t]{3}{*}{128} & Rhinocypha immaculata Selys & $\begin{array}{l}\text { Broad-winged } \\
\text { Damselfly }\end{array}$ & East Sing, West Siang \\
\hline & Order: Orthoptera & & \\
\hline & Family:Acrididae & & \\
\hline 129 & Acrida exaltata (Walker) & $\begin{array}{l}\text { Short-horned } \\
\text { Grasshopper }\end{array}$ & Changlang, Kurungkumey, Subansiri, Kane (W. Siang) \\
\hline 130 & Catantops annexus Bolivier & $\begin{array}{l}\text { Short-horned } \\
\text { Grasshopper }\end{array}$ & Changlang, Kurungkumey, Papumpare, Subansiri, Kane (W. Siang) \\
\hline 131 & $\begin{array}{l}\text { Ceracris nigricornis nigricornis } \\
\text { Walker }\end{array}$ & $\begin{array}{l}\text { Short-horned } \\
\text { Grasshopper }\end{array}$ & Subansiri, Lohit, West Siang \\
\hline 132 & Chondroacris rosea (De Geer) & $\begin{array}{l}\text { Short-horned } \\
\text { Grasshopper }\end{array}$ & Subansiri, East Siang, West Siang \\
\hline 133 & Choroedocus robustus (Serville) & $\begin{array}{l}\text { Short-horned } \\
\text { Grasshopper }\end{array}$ & Kameng, Tirap, Lohit, West Siang \\
\hline 134 & Gesonula punctifrons (Stal) & $\begin{array}{l}\text { Short-horned } \\
\text { Grasshopper }\end{array}$ & Likabali, Kambang and Kane (W. Siang) \\
\hline 135 & Holopercna darjeelingesis (Bolivar) & $\begin{array}{l}\text { Short-horned } \\
\text { Grasshopper }\end{array}$ & Subansiri, Lohit, West Siang \\
\hline 136 & Leptysma marginicollis (Serville) & $\begin{array}{l}\text { Short-horned } \\
\text { Grasshopper }\end{array}$ & New record \\
\hline 137 & Oxya hyla hyla Serville & $\begin{array}{l}\text { Short-horned } \\
\text { Grasshopper }\end{array}$ & Subansiri, East Siang, West Siang \\
\hline 138 & Oxya hyla intricate (Stal) & $\begin{array}{l}\text { Short-horned } \\
\text { Grasshopper }\end{array}$ & Likabali, Dalicamp and Kane (W. Siang) \\
\hline \multirow[t]{2}{*}{139} & Phlaeoba antennata Brunner & $\begin{array}{l}\text { Short-horned } \\
\text { Grasshopper }\end{array}$ & Subansiri, East Siang, West Siang \\
\hline & Family: Tettigoniidae & & \\
\hline 140 & Concocephalus sp. & Meadow Katydid & East Siang, Kane (W. Siang) \\
\hline \multirow[t]{2}{*}{141} & $\begin{array}{l}\text { Microcentum rhombifolium } \\
\text { (Saussure) }\end{array}$ & $\begin{array}{l}\text { Bush Katydid/ Angle } \\
\text { Wing }\end{array}$ & New record \\
\hline & Family: Gryllidae & & \\
\hline 142 & Gryllus assimilis (Fabricius) & Field Cricket & Dibang Valley, Kurungkumey, Papumpare Subansiri, Kane (W. Siang) \\
\hline 143 & Gryllus bimaculatus Dee Geer & $\begin{array}{l}\text { Black/Two-spotted } \\
\text { Cricket }\end{array}$ & $\begin{array}{l}\text { Dibang Valley, Kurungkumey, Papumpare Subansiri, Likabali and Kane } \\
\text { (W. Siang) }\end{array}$ \\
\hline
\end{tabular}




\begin{tabular}{|c|c|c|c|}
\hline & Species & Common name & Distribution / new record to Arunachal Pradesh \\
\hline & Family: Schizodactylidae & & \\
\hline 144 & Schizodactylus monstrosus (Drury) & Asian Dune Cricket & Subansiri, East Siang, Kane (W. Siang) \\
\hline \multirow[t]{2}{*}{145} & Schizodactylus tuberculatus Ander & Asian Dune Cricket & Subansiri, East Siang, Kane (W. Siang) \\
\hline & Family: Pyrgomorphidae & & \\
\hline \multirow[t]{3}{*}{146} & Tagasta indica Bolivar & Bush Hopper & Subansiri, West Siang \\
\hline & Order: Thysanoptera & & \\
\hline & Family: Thripidae & & \\
\hline 147 & Anaphothrips sudanensis Trybom & Grass Thrips & New record \\
\hline 148 & Dichromothips nakahari Mound & Orchid Thrips & New record \\
\hline 149 & Megalurothrips distalis (Karny) & Bean Thrips & Namdapha (Changlang), Kane (W. Siang) \\
\hline 150 & Megalurothrips peculiaris (Bagnall) & Flower Thrips & New record \\
\hline 151 & $\begin{array}{l}\text { Microcephalothrips abdominalis } \\
\text { (Crawford) }\end{array}$ & Composite Thrips & Namdapha (Changlang), Kane (W. Siang) \\
\hline 152 & Monilothrips kempi Moulton & Fern Thrips & New record \\
\hline 153 & Panchaetothrips indicus Bagnall & Turmeric Thrips & New record \\
\hline 154 & $\begin{array}{l}\text { Sciothrips cardamomi } \\
\text { (Ramakrishna) }\end{array}$ & Cardamom Thrips & Namdapha (Changlang). Kane (W. Siang) \\
\hline 155 & Thrips hawaiiensis (Morgan) & Cosmopolitan Thrips & New record \\
\hline \multirow[t]{2}{*}{156} & Thrips himalayanus (Pelikan) & $\begin{array}{l}\text { Himalayan } \\
\text { Terebrantia/ Thrips }\end{array}$ & New record \\
\hline & Family: Phlaeothripidae & & \\
\hline 157 & Araeothrips vamana Muraleedharan & Foliage Thrips & New record \\
\hline 158 & $\begin{array}{l}\text { Araeothrips longisetis } \\
\text { Ananthakrishnan }\end{array}$ & Foliage Thrips & Namdapha (Changlang), Kane (W. Siang) \\
\hline 159 & $\begin{array}{l}\text { Dolichothrips montanus } \\
\text { Ananthakrishnan }\end{array}$ & Thrips & New record \\
\hline 160 & Elaphrothirps spiniceps Bagnall & Litter Thrips & New record \\
\hline 161 & Gigantothrips elegans Zimmerman & Ficus Thrips & New record \\
\hline 162 & Haplothrips gowdeyi (Franklin) & Thrips & New record \\
\hline 163 & Haplothrips tenuipennis Bagnall & Thrips & New record \\
\hline 164 & Liothrips ananthakrishnani Sen & Gall Thrips & Tawang, Kane (W. Siang) \\
\hline 165 & $\begin{array}{l}\text { Liothrips himalayanus } \\
\text { Ananthakrishnan \& Jagadish }\end{array}$ & Gall Thrips & Papum Pare, Kane (W. Siang) \\
\hline 166 & Liothrips sp. & Gall Thrips & East Siang, Kane (W. Siang) \\
\hline 167 & Meiothrips menoni Ananthakrishnan & Mycophagous Thrips & New record \\
\hline 168 & Nesothrips lativentris (Karny) & Litter Thrips & Namdapha (Changlang), Kane (W. Siang) \\
\hline 169 & $\begin{array}{l}\text { Neoheegeria montana } \\
\text { Ananthakrishnan and Jagadish }\end{array}$ & Thrips & New record \\
\hline 170 & $\begin{array}{l}\text { Xylaplothrips debilis } \\
\text { Ananthakrishnan \& Jagadish }\end{array}$ & Litter Thrips & Namdapha (Changlang), Kane (W. Siang) \\
\hline
\end{tabular}

Anonymous, Arunachal Pradesh Human Development Report 2005. Department of Planning, Government of Arunachal Pradesh, Publication.

Ghate, H.Y., L. Borowiec, N.S. Rane, S.P. Ranade \& S. Pandit (2003). Tortoise beetles and their host plants from Pune (Maharashtra state, India) and nearby places (Coleoptera:
Chrysomelidae: Cassidinae). Genus, Wroclaw 14: 519-539.

Ghosh, A.K. (1985). Namdapha Biosphere Reserve-An overview. Records of Zoological Survey of India 82 (1-4): 1-8.

Ghosh, A.K. \& T. Sengupta (1982). Insect Collection, Preservation and Study (Handbook). Zoological Survey of India Publication, 65pp. 
Lahiri, A.R. (1979a). Odonata (Insecta) from different states of northeastern India. Oriental Insects 13(1-2): 119-132.

Lahiri, A.R. (1979b). New records of Odonata from Tripura and Arunachal Pradesh. Proceeding of the Zoological Society (Calcutta) 30(1-2): 45-49.

Mitra, A. (2006). Current status of the odonata of Bhutan: A checklist with four new records. Bhu. J. RNR. 2(1): 136-143.

Mitra, S.K. (1976). Some Collembola from Arunachal Pradesh (Entomobryidae). Oriental Insects 10: 143-149.

Myer, N., R.A. Muttermeir, C.A. Muttermeir, A.B. Da Fonseca \& J. Kent (2000). Biodiversity hot spots for conservation priorities. Nature 403: 853-858.

Pal, T.K. (1985). A revision of Indian Psammoecus Latreille (Coleoptera: Silvanidae). Miscellaneous Publication, Records of Zoological survey of India. Occasional paper 71: 1-54.

Pal, T.K. (1992a). On a collection of Clavicorn beetles (FamilyErotylidae, Endomychidae and Languriidae) from Arunachal Pradesh, India. Records of Zoological Survey of India 90(14): $203-210$

Pal, T.K. (1992b). Inopelidae and Elacatidae (Coleoptera: Heteroptera) from Arunachal Pradesh, India. Records of Zoological survey of India 91(3-4): 343-351.

Pal, T.K. (1992c). A new inopeplid species (Coleoptera: Cucujoidae) from Patkoi Hill Range of Arunachal Pradesh Journal of Bombay Natural History Society 89(2): 238-241.

Pal, T.K. (1993). On a collection of Passalidae and Lucanidae (Insecta: Coleoptera) from Arunachal Pradesh India. Records of Zoological survey of India 90 (1-4): 135-145.
Ramakrishna \& J.R.B. Alfred (2006). Fauna of Arunachal Pradesh: an overview, pp. 1-20. In: Editor-Director. Fauna of Arunachal Pradesh. State fauna Series 13 (Part-I). Zoological Survey of India Publication, 666pp.

Sen, S., N.K. Pramanik \& C.K. Sengupta (1998). Thysanoptera fauna of northeastern India. Records of Zoological Survey of India - Occasional Paper 100: 1-123.

Sengupta, C.K. \& T. Sengupta (1981). Cerambycidae (Coleoptera) of Arunachal Pradesh. Records of the Zoological Survey of India 78(1-4): 133-154.

Singh, T.P., S. Singh, P.S. Roy \& B.S.P. Rao (2002). Vegetation mapping and characterization in West Siang District of Arunachal Pradesh, India- a satellite remote sensing based approach. Current Science 83(10): 1221-1230.

Subramanian, K.A. (2005). Dragonflies and Damselflies of Peninsular India - A Field Guide. E-Book of Project Lifescape. Centre for Ecological Sciences, Indian Institute of Sciences and Indian Academy of Sciences, Bangalore, India, 118pp.

Tandon, S.K. \& S. Khera (1978). Ecology and distribution of grasshoppers (Orthoptera: Acridoidea) in Arunachal Pradesh, India and the impact of human activities on their ecology and distribution. Memoirs of the Entomology St. John's College 6: 73-91

Singh, O.T., J. Chakravorty, S. Nabom \& D. Kato (2007). Species diversity and occurrence of edible insects with special reference to Coleopterans of Arunachal Pradesh. Journal of Natural Conservation 19(1): 167- 176. 